\title{
RACIONALIDAD INDIVIDUAL Y COLECTIVA: MECANISMOS ECONÓMICOS Y JURÍDICOS DE ARTICULACIÓN ${ }^{1}$
}

\section{Introducción}

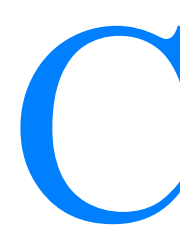

omo economista en un foro eminentemente jurídico me propongo aquí un triple objetivo. Primero, exponer el punto de vista y los conceptos básicos con los que los economistas analizamos el papel del Estado en la economía y la determinación del tamaño deseable del Sector Público, tema que constituye la cuestión de fondo de estas Jornadas sobre Neoliberalismo y Estado. Frente a posturas doctrinales y al ondear de las banderas ideológicas, la moderna teoría económica señala con claridad las vías prometedoras y los caminos prohibidos para un análisis serio de la cuestión.

Segundo, dado el carácter pluridisciplinar del acontecimiento, no estará de más aprovechar la ocasión para ilustrar los principios de procedimiento de los economistas. Mediante una drástica reducción del universo de discurso en el que la racionalidad de los individuos adopta una forma extremadamente simple y axiomatizada, el economista teórico se plantea cuestiones perfectamente definidas que tienen una respuesta no ambigua. Cuestiones que a pesar de su aparente sencillez reflejan los problemas más profundos en la interacción entre individuos independientes y que bastan para generar las grandes perplejidades de la problemática económica. Esta es la metodología fundamental que ha hecho de la economía una ciencia cuyo desarrollo ha adoptado la forma de una sucesión de modelos de complejidad creciente tan característica de los programas de investigación de las ciencias maduras. La formalización ha dado rigor a la teoría y vitalidad a la sucesión de modelos. Sin embargo, las mismas características que hacen de la ciencia económica un instrumento

${ }^{1}$ Department d'Economia, Universitat Pompeu Fabra, Barcelona. Este trabajo ha sido financiado por la DGICYT. 
extremadamente afilado para diseccionar la realidad social, justifican la recomendación vehemente de que un instrumento tan cortante no debe dejarse nunca en manos inexpertas o malintencionadas. Somos los propios economistas los que debemos impedir extrapolaciones no justificadas en la interpretación de los resultados formales.

Tercero, intentaré -con prudencia, eso sí- aventurarme en el campo de la ciencia jurídica y sugerir algunos temas en los que las técnicas de análisis de los economistas puedan ser de alguna utilidad. En particular, ilustraremos cómo un modelo tomado a préstamo de los economistas puede ser útil para analizar, desde una óptica prescriptiva, el diseño legislativo como un mecanismo de articulación entre la racionalidad individual y la colectiva.

\section{Los mecanismos económicos y la información}

El punto de partida de la visión del economista es que las partículas elementales del universo económico son los individuos que interaccionan entre sí mediante relaciones de intercambio. Las acciones de los individuos consisten básicamente en elegir una de entre muchas alternativas posibles. Se supone que el principio de racionalidad informa esa conducta, en el sentido de que dicha elección se efectúa de acuerdo con unas preferencias que ordenan todas las alternativas imaginables. De este modo, se dice que una conducta es racional si existe un orden de preferencias que explique las elecciones del individuo. La «racionalidad» o el origen de las preferencias mismas no se cuestiona. Cada individuo, en tanto que «homo economicus», queda pues caracterizado por unos recursos iniciales (es decir, un conjunto de bienes), un orden de preferencias y una tecnología que determina sus posibilidades de transformar unos recursos en otros.

Si tanto los objetivos (satisfacer las necesidades) como las restricciones impuestas por la naturaleza (las restricciones tecnológicas y la limitación de los recursos iniciales) están bien definidos el problema de la racionalidad económica puede reducirse a un sencillo problema de maximización condicionada. La filiación individualista de estos planteamientos es clara.

Sin embargo, la racionalidad de la conducta individual es la parte más trivial de lo económico. El guiso empieza a adquirir sustancia cuando se considera que la división del trabajo aumenta la productividad, lo cual implica la especialización en la producción. Por otra parte, las preferencias de los consumidores son tales que se prioriza siempre la diversificación del consumo. La especialización en la producción y la diversificación en el consumo conducen inevitablemente 
a la necesidad del intercambio. Aparece aquí con todo su esplendor la dimensión social de lo económico.

Estas consideraciones sitúan el problema en un nivel distinto desde el momento en que toda relación de intercambio, y en general todas las relaciones sociales, son el resultado de la interacción de múltiples racionalidades individuales distintas. Se plantea entonces la cuestión de hasta qué punto se puede hablar de racionalidad colectiva y en qué medida se relaciona ésta con la racionalidad individual.

En su célebre manual, Samuelson afirma que la primera lección que debe aprenderse de Economía es que, con frecuencia, las cosas no son lo que parecen. Y pone un énfasis especial en advertir de los peligros de la falacia de la composición, que consiste en predicar del todo lo que sólo es cierto de la parte. Ciertamente, una manera de mantener limpia una ciudad es que cada ciudadano mantenga limpia la parte de la calle que le corresponde. La extrapolación de lo particular a lo colectivo funciona en este caso. Pero en muchos otros no y la articulación entre lo individual y lo colectivo es mucho más sutil e inesperada aunque no por ello deja de ser predecible. La predicción es posible pero es contraintuitiva. Si, por ejemplo, en un espectáculo público en la calle tenemos dificultades para ver lo que pasa, podemos ponernos de puntillas y mejorar la situación significativamente. Pero si todos deciden hacer lo mismo, y adoptan la conducta óptima desde un punto de vista individual, el resultado final es desastroso: todos siguen viendo el espectáculo tan mal como antes pero tienen que soportar la incomodidad adicional que resulta de mantenerse de puntillas.

Hemos definido la racionalidad individual como la simple adecuación de la conducta a unos intereses o prioridades coherentes. De modo análogo podríamos hablar de racionalidad social siempre que la conducta del sistema entero fuera adecuada a un sistema de prioridades bien establecido. Se plantea entonces el problema de cómo construir un sistema de prioridades sociales a partir de prioridades individuales. Este tema, conocido como agregación de preferencias, ha sido el centro de atención fundamental de la teoría de la elección social. Y como se indica en el trabajo de Herrero existen serias dificultades para construir consistentemente una racionalidad social a partir de la racionalidad individual.

El problema que nos interesa analizar aquí se sitúa en un plano de análisis posterior. Se trata de examinar desde un punto de vista prescriptivo los mecanismos de coordinación que permiten realizar unas preferencias sociales, cuyo origen o justificación no se cuestiona. Esta es la perspectiva que $\operatorname{Sen}^{2}$ denomina «ingenieril» y consiste

\footnotetext{
${ }^{2}$ Véase Sen, On ethics and economics, Basil \& Blackwell, 1987.
} 
en determinar los procesos de decisión que permiten alcanzar una situación social deseable de acuerdo con un criterio de bienestar social dado. Hemos solventado así, de un plumazo, toda la Teoría de la Elección Social.

La decisión social óptima varía según cuál sea el entorno económico (hay una acción óptima para cada situación). Si conociéramos los datos sobre la situación (es decir, la información acerca de las preferencias, la tecnología y los recursos iniciales) el problema se reduciría de nuevo a un problema técnico de maximización. Pero un elemento adicional complica la situación: la información sobre la economía está dispersa entre los agentes. La adopción de un plan conjunto coherente requiere el intercambio de información.

Un mecanismo económico es un modelo que describe los procedimientos adoptados por una comunidad de individuos para coordinar sus acciones. En particular debe describir los flujos de información entre los agentes y el uso que éstos hacen de ellos para tomar sus decisiones. Transmitir y procesar información es muy costoso. Por otra parte, es muy posible que los individuos no estén dispuestos a proporcionar la información exigida por un mecanismo. Por ello es una característica importante de los mecanismos el minimizar la información, de un lado, y proporcionar incentivos para la revelación correcta de los datos por parte de los agentes económicos, de otro.

\section{La mano invisible y los fallos del mercado}

A principios de la década de los sesenta Arrow y Debreu demostraron la existencia y eficiencia del equilibrio competitivo en un celebrado trabajo en el que demostraban rigurosamente las condiciones bajo las cuales es cierta la conjetura de la Mano Invisible de Adam Smith. En ausencia de bienes públicos, efectos externos y rendimientos crecientes, si existen muchos agentes compradores y vendedores y un mercado para cada bien en el que se forma un precio competitivo conocido por todos, el sistema de precios proporciona información suficiente acerca de los datos de la economía para que las decisiones descentralizadas e interesadas de todos los agentes económicos conduzcan a través de los mecanismos de la oferta y la demanda a un estado económico caracterizado por la eficiencia (en el sentido de Pareto) en la asignación de los recursos.

Dos son las consideraciones básicas que no permiten interpretar este resultado como una apología del liberalismo económico. En primer lugar, el concepto de eficiencia económica u óptimo de Pareto es totalmente insensible a las consideraciones distributivas y por 
tanto no está claro que sea un objetivo social legítimo ${ }^{3}$. En segundo lugar, el resultado de Arrow y Debreu no demuestra la superioridad del mecanismo de mercado competitivo, sino que establece rigurosamente las condiciones bajo las cuales tal pretensión está justificada. Desafortunadamente, estas condiciones no son tan generales como sería de desear y existen muchos casos en los que se produce una ruptura radical entre lo individual y lo colectivo. Veamos algunos ejemplos sencillos en los que aparece esta brecha entre la racionalidad individual y la colectiva.

\section{1. Los bienes públicos}

En Economía, se llama bien público a aquel que es susceptible de un consumo colectivo. Una patata no es un bien público porque el acto de consumo por parte de A impide que B pueda consumirla. Por el contrario, una emisión de radio es un bien público puesto que millones de individuos pueden disfrutarla simultáneamente. Según esta definición, que un bien sea público o no depende de su naturaleza y no del tipo de organización de la cual se haya dotado la sociedad considerada.

Supongamos que tres individuos A, B y C valoran una emisión de radio en 10, 6 y 5 pesetas respectivamente. Una emisión de radio vale 12 pesetas. No hacer la emisión es ineficiente. En efecto, existe una asignación alternativa -que consiste en pagar cuatro pesetas cada uno- que significa un beneficio de seis pesetas para A, dos para B y una para C: todos están mejor que antes. Poseyendo toda la información es fácil determinar lo que es ineficiente. Pero supongamos ahora que la valoración de cada individuo es información privada de modo que nadie sabe la valoración del vecino. Conociendo que su declaración acerca de la valoración subjetiva del bien público puede afectar a su contribución a la financiación del mismo, el individuo $\mathrm{C}$ puede argüir que no le interesa el programa de radio.

${ }^{3}$ Un estado económico es eficiente (óptimo en el sentido de Pareto) si es imposible mejorar a algún individuo sin perjudicar a nadie. Por consecuencia, si una asignación de recursos es ineficiente, será posible encontrar una asignación alternativa que sea unánimemente preferida a dicha asignación. Dicho de esta forma parece difícil oponerse al criterio de unanimidad implícito en la noción de eficiencia. Piénsese, no obstante, que si tenemos cuatro manzanas a repartir entre A y B y se las damos todas a A alcanzaremos una asignación eficiente (para mejorar a $\mathrm{B}$ es inevitable quitar una manzana a A, lo cual le perjudica). En realidad todas las distribuciones que repartan todas las manzanas son eficientes. No todas ellas serán justas. Las distribuciones ineficientes son aquellas que no reparten todas las manzanas: siempre será posible repartir la manzana no distribuida y alcanzar una situación unánimemente preferida a la inicial. 
Con todo, los individuos A y B pueden acordar, por ejemplo, pagar siete y cinco pesetas respectivamente, con lo que cubrirán el coste del programa y obtendrán beneficios de tres $\mathrm{y}$ una pesetas respectivamente. Pero $\mathrm{C}$ también puede disfrutar de la emisión del programa sin que se note, obteniendo un beneficio de cinco pesetas: ¡sale a cuenta simular una valoración inferior a la real! Si esto es cierto, todos tratarán de simular indiferencia $\mathrm{y}$, si todos lo hacen, el bien público acabará por no producirse. En efecto, con la información disponible (que no es la real) la decisión óptima es no hacerlo.

\subsection{El dilema del prisionero}

Dos delincuentes han sido detenidos. El juez sabe con certeza que son culpables de un crimen por el que se les puede condenar a diez años de cárcel a cada uno. Pero no tiene pruebas suficientes y sin una confesión de uno de ellos lo máximo que puede imponerles es cuatro años de cárcel. Decide entonces ponerlos en celdas aisladas y ofrecerles lo siguiente: la pena que les va a corresponder dependerá del comportamiento de ambos: si los dos confiesan ocho años a cada uno (de hecho tiene pruebas suficientes para condenarles a diez). Si uno confiesa y el otro no, máxima pena para el primero y mínima para el segundo. Si ninguno de los dos confiesa no tiene más remedio que ponerles cuatro años por falta de pruebas. Las consecuencias de las estrategias respectivas de confesar, C, y no confesar, N, se representan esquemáticamente en la tabla adjunta.

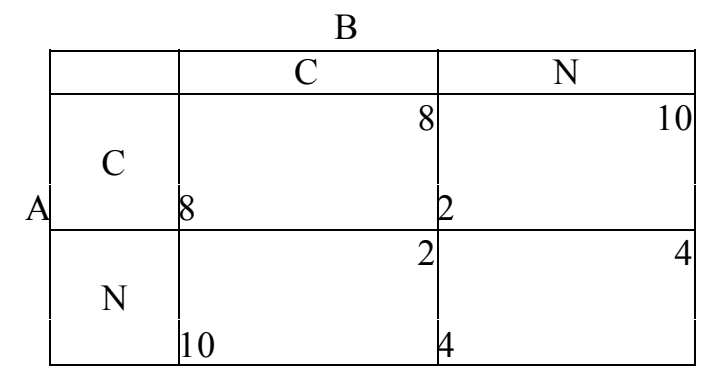

Figura 1: El dilema del prisionero.

Obsérvese que en este caso, contrariamente a lo que ocurre en la decisión de un consumidor competitivo al comprar una bolsa de patatas a un precio dado, ningún individuo puede conocer exactamente las consecuencias de su acción, puesto que el resultado depende también 
de lo que haga el otro. Esta interdependencia estratégica hace mucho más difícil definir qué significa conducta racional en este contexto. Afortunadamente, en este ejemplo las cosas son bastante sencillas puesto que para ambos jugadores confesar es una estrategia dominante. En efecto, si B ha decidido confesar A se encuentra con que debe escoger entre ocho años (si también confiesa) o diez años ( si a pesar de todo prefiere no confesar). Está claro que preferirá confesar. Pero si $\mathrm{B}$ ha decidido no confesar debe escoger entre dos años (si confiesa) o cuatro años (si no confiesa). También en este caso A prefiere confesar. Por lo tanto si B confiesa como si no, la mejor respuesta de A es confesar. Si ambos confiesan, el resultado de la conducta racional individual es que pasarán ocho años en la cárcel. Pero éste es un resultado ineficiente ${ }^{4}$ : si en vez de perseguir cada uno su propio interés hubiesen pactado no confesar hubieran conseguido salir con sólo cuatro años, situación que es unánimemente preferida. Y aunque pacten, una vez seguros de que el acuerdo se va a cumplir, la tentación de que cualquiera de ellos decida traicionar el acuerdo y reducir la pena a sólo dos años es casi irresistible.

Existen muchas situaciones económicas interesantes que tienen la misma estructura formal que el dilema del prisionero: dos empresas o países que compiten en el mercado de un mismo producto se ven forzadas por la lógica de la maximización del beneficio privado a una reducción de precios que les conduce inexorablemente a niveles de beneficios muy inferiores. Aquí no hay mano invisible que armonice la racionalidad individual y el interés colectivo. Todo lo contrario, se necesita una mano muy visible que corrija el funcionamiento espontáneo de los individuos guiados por su oportunismo.

\subsection{El modelo de Hotelling}

Supongamos que dos vendedores de helados venden un producto uniforme al mismo precio. En una playa de 600 metros de longitud hay un individuo en cada metro tomando alegremente el sol y dispuesto a comerse un helado. Como los dos vendedores son indistinguibles lo comprará al vendedor que le caiga más cerca. Su objetivo es minimizar la distancia. Los vendedores deben escoger dónde ubicarse en la playa. Su objetivo es conseguir el número máximo de clientes.

¿Cuál es la ubicación socialmente óptima? Afortunadamente en este caso no hay duda. Si el primer vendedor se sitúa en el metro 150

${ }^{4}$ Obsérvese que aquí el resultado es ineficiente desde el punto de vista de las preferencias de los dos jugadores (es decir, de los delincuentes). 
(el punto A de la figura) y el segundo en el 450 (el punto B de la figura) resultará que el punto $\mathrm{C}$ separa a los clientes de ambos. De este modo obtendrán 300 clientes cada uno. Por otra parte ningún consumidor deberá andar más de 150 metros para conseguir un helado.

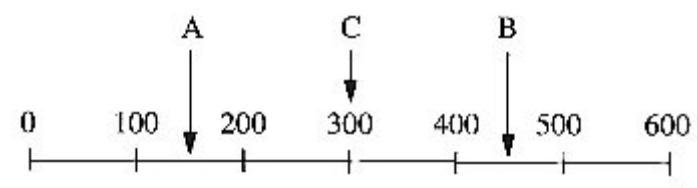

Figura 2: La playa de Hotelling.

¿Qué ocurrirá si el primer vendedor decide ubicarse en el punto 150, que es el que le corresponde en el óptimo social? Pues sencillamente que si el segundo vendedor se coloca en el punto 151 conseguirá 450 clientes (todos los que quedan a la derecha del 150). No cabe duda de que no va a renunciar a una oportunidad tan jugosa. El primer vendedor se da cuenta de que cualquiera que sea el punto que escoja, el segundo vendedor se colocará junto a él, pero decantado hacia el «lado largo» del mercado. Anticipándose a su conducta, el único modo de evitar esta apropiación es escoger una ubicación en la que no haya lado largo, a saber, el punto 300. El razonamiento del segundo vendedor será idéntico y tendremos que el resultado de las decisiones independientes de los dos vendedores guiados por sus intereses privados ha conducido a una situación absurda desde el punto de vista social en la que los vendedores están situados el uno junto al otro en el centro de la playa, siguen repartiéndose los clientes a partes iguales, pero algunos consumidores tienen que andar 300 metros para consumir su helado. Los intereses individuales han conducido a un estado social subóptimo. Si en vez de consumidores distribuidos uniformemente a lo largo de una playa pensamos en votantes distribuidos a lo largo del espectro político podemos explicar la tendencia de los partidos políticos a ubicarse en el centro.

\section{Los fallos del Estado}

Desgraciadamente, cuando se producen fallos del mercado la mera transferencia de la responsabilidad al gobierno no soluciona los problemas. La intervención del gobierno supone diseñar un nuevo mecanismo de asignación de recursos en el que interviene un agente adicional que, al igual que los demás, va a tener problemas de recopilación y procesamiento de información. No está claro cómo la 
introducción de este nuevo agente podrá sortear los problemas que dieron luz a los fallos de mercado. Este ha sido el drama del moderno Estado de Bienestar: la decidida voluntad política de intervenir en el sistema social y las sólidas justificaciones teóricas, éticas y prácticas para hacerlo no se han visto acompañadas por un esfuerzo paralelo en articular los mecanismos económicos específicos a través de los cuales poner en práctica esta intervención.

El diseño de un mecanismo no se reduce al mero enunciado de los grandes objetivos sociales que se pretende alcanzar. Hay que indicar sobre qué información y sobre qué criterios se basan las decisiones concretas que hay que adoptar en cada situación. Al directivo de una empresa pública no basta con indicarle que debe mejorar la situación del país. Hay que indicarle si debe utilizar los precios de mercado de los factores productivos para computar los costos y si debe minimizarlos o, por el contrario, debe priorizar la utilización de ciertos factores productivos (si lo que se pretende es reducir el desempleo) aunque ello suponga incrementar los costes o incluso incurrir en pérdidas. El veredicto final sobre un mecanismo económico se basará siempre en los resultados para el entero sistema social de la interacción de los comportamientos prescritos a los distintos agentes. No basta con analizar las consecuencias a corto plazo para un solo agente. Como ya señalamos al hablar de la falacia de la composición, ocurre muy a menudo que la presencia de múltiples agentes con intereses diversos y su interacción estratégica conduce a resultados totalmente inesperados, y un cierto tipo de actuación que en principio parece plausible y deseable tiene efectos contraproducentes.

El gobierno, en tanto que agente económico, tiene una característica peculiar que le distingue de los demás agentes y le abre muchas posibilidades: está investido de poderes coactivos. Consecuentemente, la clase de mecanismos posibles se amplía considerablemente. Ello permite la consideración de una nueva clase de mecanismos que posibilitan la existencia de intercambios no voluntarios. Sin embargo, es esa misma posibilidad la que aumentará los incentivos de los individuos a ocultar las características no observables.

Como las decisiones coactivas tienen poderosos efectos redistributivos, existen mecanismos de control para proteger los derechos de los agentes privados que explican la burocratización y falta de flexibilidad que caracterizan las actuaciones de las administraciones públicas. Se trata pues de un arma de doble filo: los poderes coactivos de uno de los agentes amplían considerablemente la clase de mecanismos posibles, pero los instrumentos de control que tales poderes exigen para evitar abusos dan lugar a las graves limitaciones señaladas por Stiglitz ${ }^{5}$

${ }^{5}$ J. E. Stiglitz, The Economic Role of the State, Basil Blackwell, 1989. 
que ponen en tela de juicio la creencia indiscriminada en el Sector Público como alternativa indiscutible al mercado.

Las actuaciones del Sector Público en el mercado de trabajo se caracterizan por la uniformidad salarial y el carácter vitalicio de los contratos. En general, tenderá a dar un peso excesivo a los criterios de equidad y uniformidad. La actividad de la administración induce a la aparición de «cazarrecompensas»-que se limitan a explotar la discrecionalidad de la administración pública no sometida a los criterios de mercado y viven de subvenciones y contratos especiales- y hace rentables actividades que consisten exclusivamente en saltarse la ley -como es el caso de los contrabandistas-. Pero las disfunciones más comunes son algo más sutiles y por ello menos obvias. Toda intervención del gobierno genera una reacción de los individuos que a menudo conduce a situaciones muy distintas a las inicialmente previstas. Veámoslo con un sencillo ejemplo numérico en el cual, en un contexto tan complejo y múltiples como el de las modernas economías de mercado, el excesivo celo redistributivo genera ineficiencias y desigualdades no queridas.

Consideremos el problema de la fijación de tarifas de un servicio público como el correo. En la tabla adjunta se indican los costes y el número de usuarios de cada servicio postal. Dado que los costes unitarios son distintos, una alternativa posible es fijar el precio de cada servicio de acuerdo con su coste. Sin embargo, ello significa que los campesinos, que son precisamente una de las clases sociales menos favorecidas, tienen que pagar precios muy altos.

\begin{tabular}{|l|c|c|c|c|}
\hline & Interurbano & Rural & Urbano & Total \\
\hline Coste unitario.......... & 30 & 50 & 10 & \\
Número de usuarios. & 10 & 10 & 40 & 60 \\
Costes totales.......... & 300 & 500 & 400 & 1.200 \\
\hline
\end{tabular}

Una idea bastante común consiste en usar la financiación del servicio público con propósitos redistributivos. Si se establece un precio único de 20 pesetas por servicio postal -del tipo que sea- se obtienen unos ingresos de 1.200 por los 60 servicios, lo cual cubre exactamente los costes totales de los mismos, tal como se indica en la última columna del cuadro. Se produce entonces un subsidio encubierto: los ricos habitantes de las ciudades están financiando los servicios postales de las pobres zonas rurales, pagando 20 pesetas por un servicio cuyo coste real es de 10 pesetas. Sin embargo, el afán redistributivo abre una vía de actuación al sector privado y acaba conduciendo a una situación que no tiene 
ninguna justificación ética ni económica. Si una compañía de mensajeros se percata de la situación puede ofrecer servicios urbanos (que le cuestan 10 pesetas) a 15 pesetas, lo que le producirá unos beneficios de 200 pesetas, que es lo mismo que ahorrarán los usuarios urbanos. La situación del servicio público se deteriorará rápidamente: en vez de las 1.200 pesetas esperadas de ingresos obtendrá 400 pesetas e incurrirá en unos costes (los rurales más los interurbanos) de 800 , lo cual significará unas pérdidas de 400 pesetas. A la larga no tendrá más remedio que cargar un precio de 40 pesetas, que es el que le permite equilibrar el presupuesto tras la pérdida de los usuarios urbanos. Ahora son los usuarios interurbanos quienes soportan el subsidio a las zonas rurales: el afán redistributivo ha desembocado en un beneficio extraordinario para una empresa privada de mensajería y en una carga excesiva sobre los pocos usuarios interurbanos.

Llegamos a una primera conclusión: como consecuencia de las inevitables reacciones estratégicas de los demás agentes, las directrices básicas que hay que dar a los agentes decisores del Sector Público deben ir mucho más allá de la mera afirmación de los grandes objetivos sociales que hay que alcanzar. Pero eso no es todo. A menudo se piensa en el gobierno como un agente único y dotado de una racionalidad propia. Sin embargo, al igual que ha sucedido con la empresa, los economistas teóricos han dejado de contemplar al gobierno como una unidad de simple decisión para visualizarlo como una forma organizativa alternativa que regula y coordina las decisiones de múltiples agentes con informaciones y objetivos diversos. Su funcionamiento interno se caracteriza por unas relaciones jerarquizadas que normalmente no están reguladas por precios. Será de interés estudiar la descentralización de las decisiones, los flujos de información, el diseño de la estructura jerárquica, y la elaboración de estructuras de remuneración que generen los incentivos apropiados para hacer coincidir en la medida de lo posible los objetivos individuales con los objetivos de la organización y garanticen el cumplimiento de los objetivos sociales. Mercado, empresa y administraciones públicas son formas alternativas de organización dirigidas a la solución del problema de la asignación de recursos. Pero el discurso actual de los economistas hoy no es tanto un debate sobre el tamaño relativo de cada una de estas formas organizativas consideradas como productos acabados, como un esfuerzo riguroso por diseñar estructuras organizativas alternativas para la empresa y la administración pública que permitan transmitir eficientemente la información, coordinar eficazmente las decisiones independientes y canalizar los intereses individuales en la dirección de los colectivos. 


\section{Economía y Derecho}

En el modelo de Arrow-Debreu se analizan el funcionamiento y las propiedades de un mercado competitivo en el supuesto de que todos los empresarios maximizan los beneficios. Buena parte de la formación de los estudiantes de Economía consiste en enseñarles a hacer precisamente lo que allí habíamos supuesto.

Análogamente, la formación de los estudiantes de Derecho consiste básicamente en saber, dada una acción concreta de un individuo, qué debe hacer el juez para aplicar correctamente el Derecho. Se trata ante todo de conocer para aplicar. Idealmente el fallo no es más que una consecuencia lógica de las premisas, que son los fundamentos de Derecho, de un lado, y los antecedentes de hecho, de otro. La realidad, no obstante, es considerablemente más compleja y multitud de dificultades se interponen en el camino del estudioso. No hay hechos en bruto sino hechos interpretados a través de normas. Nuestro ordenamiento jurídico está plagado de lagunas, inconsistencias y ambigüedades. Todo ello hace que el trabajo de un juez incorpore decisiones y que realizar este trabajo correctamente sea algo extremadamente delicado y difícil. En ello reside la sutileza que requiere la formación del jurista.

Pues bien, al igual que en la Teoría Económica del Bienestar se supone que todo el mundo hace perfectamente lo que tanto cuesta enseñar, voy a empezar haciendo la heroica hipótesis de que el sistema normativo tiene las propiedades de plenitud y consistencia, de un lado, y que los jueces aplican correctamente el Derecho en todo caso. En un sistema de tal naturaleza a cada acción le corresponde una, y sólo una consecuencia, de modo que todo el mundo sabe a qué atenerse y la seguridad jurídica es total.

Vamos a situamos ahora en un contexto muy abstracto y vamos a describir una sociedad como un conjunto de $\mathrm{n}$ individuos, que toman decisiones acerca de qué hacer. Sea $a_{i}$ la acción del individuo $i$ y $a=\left(a_{1}, a_{2}, \ldots, a_{n}\right)$ la acción conjunta de todos los individuos. Un estado social es una descripción de la situación de cada uno de los individuos. Denotaremos por $s_{i}$ a la situación del individuo $i$ y por $s=\left(s_{1}, s_{2}, \ldots, s_{n}\right)$ al estado social que describe la situación de todos los individuos.

Los individuos, en el ejercicio de sus libertades eligen sus acciones, lo cual hace que se pase de un estado inicial $s$ a otro $s^{\prime}$. De este modo, el efecto conjunto de las acciones de los agentes determina el estado social final. Denotemos por $\Phi:(a ; s) \rightarrow s^{\prime}$ a la función que describe cómo el estado final $s$ ' depende del estado inicial $s$ y de las acciones de todos los individuos.

Vamos a suponer que los individuos tienen sus preferencias, no sólo sobre los distintos estados sociales, sino que tienen en cuenta 
las acciones que los han originado de modo que, dados dos pares $(a, s)$ y $\left(a^{\prime}, s^{\prime}\right)$, siempre pueden decir cuál de ellos prefieren. Si prefieren el primer par al segundo escribiremos $(a$, s) $P_{i}\left(a^{\prime}, s^{\prime}\right)$, donde $P_{i}$ es una relación binaria que expresa las preferencias del individuo $i$. Obsérvese que no hacemos ninguna hipótesis acerca de estas preferencias y en particular no postulamos las razones por las cuales se prefiere una acción a otra: puede ser por motivos económicos, éticos, altruistas o religiosos.

La segunda hipótesis que voy a postular es que la sociedad en su conjunto también tiene unas preferencias sobre los pares $(a, s)$. Con esto obviamos el análisis de las decisiones institucionales colectivas ${ }^{6}$ y el problema de determinar la posible relación que tienen con las preferencias individuales. Estas «preferencias sociales además de recoger de alguna $\mathrm{u}$ otra forma las preferencias de las mayorías (es decir, un modo de agregar preferencias), de promover una sociedad próspera y criterios generales de eficiencia y justicia distributiva ${ }^{7}$ deben incluir también valores fundamentales de libertad, igualdad y justicia. En particular se ponen límites al ejercicio de las libertades individuales a través de derechos individuales fundamentales.

Un sistema jurídico prohíbe cierto tipo de acciones pero los individuos pueden escoger llevarlas a cabo. Sin embargo, un sistema jurídico altera la relación que existe entre acciones y consecuencias y, de este modo, influye sobre las acciones. Si la ley prohíbe aparcar en un determinado lugar puedo hacer caso omiso y seguir aparcando pero la consecuencia es que (con una cierta probabilidad) tendré que pagar una multa.

La estructura del problema es semejante al planteamiento de los mecanismos descentralizados de asignación de recursos en el siguiente sentido. La realización de las preferencias sociales -que recogen las preferencias y respetan los derechos básicos de los individuos de la sociedad- se representa mediante la función $\sigma$ que prescribiría en cada caso las acciones que hay que llevar a cabo para pasar de un estado social a otro. Pero en nuestras sociedades los mecanismos de decisión son descentralizados, por razones informacionales, pero también por razones más fundamentales: un ingrediente básico de estas preferencias colectivas consiste en el establecimiento de ciertos límites a las decisiones que puede tomar la sociedad en contra de intereses valiosos de los individuos. Como señala Caracciolo, «la protección consiste básicamente en conceder, como

${ }^{6}$ Véase por ejemplo, R. Caracciolo, «Derecho y decisiones colectivas», Revista del Centro de Estudios Constitucionales, N. ${ }^{\circ} 10,1991$, pp. 25-48.

${ }^{7}$ Soy expresamente vago en todos estos aspectos: una discusión detallada nos llevaría demasiado lejos. Baste decir aquí que éstos son aspectos que los economistas han incorporado sistemáticamente en su análisis de las preferencias sociales. 
condición mínima, el control personal y exclusivo sobre determinados tipos de comportamiento». Cada individuo escoge de modo independiente su acción preferida $a_{i}$. Pero las consecuencias de su acción dependen de las acciones de los demás individuos, de un lado, y del sistema jurídico, de otro.

Dotar de contenido a un planteamiento tan abstracto es labor ciertamente complicada. Pero podemos ya plantearnos formalmente algunas cuestiones de interés. En la transición de un estado social a otro los individuos toman libremente sus acciones Controlan pues la función $\gamma$ representada en la figura 2. A través del sistema jurídico, la sociedad controla la función $\varphi$ que transforma acciones en consecuencias. Al tomar sus decisiones los individuos tienen en cuenta el estado inicial, sus preferencias subjetivas y las consecuencias de sus acciones (lo cual significa que tienen en cuenta el sistema jurídico).

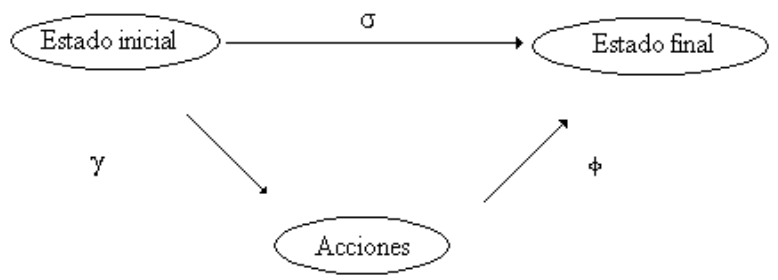

Dadas unas preferencias sociales (es decir, un sistema de valores que prioriza alternativas teniendo en cuenta criterios económicos, de justicia, derechos individuales básicos, etc.), se plantean entonces dos grandes cuestiones:

a) En qué medida el sistema jurídico actual -representado por una función de realización $\varphi$ que transforma acciones en consecuencias- induce a los individuos a actuar conforme a las preferencias sociales.

b) ¿Existe algún sistema jurídico que mejore el funcionamiento del actual, en el sentido de que conduzca a estados sociales más deseables?

El sistema jurídico se constituye fundamentalmente en un mecanismo de articulación entre la racionalidad individual y la colectiva, el modo de conseguir ciertos objetivos sociales de progreso económico y social, ejercicio de libertades y respeto a los derechos básicos del individuo. El modelo abstracto que hemos planteado nos 
permite contemplar el sistema jurídico como un mecanismo para canalizar los intereses individuales en la dirección de los objetivos que la sociedad se ha marcado. Lo hemos conseguido haciendo abstracción de toda la problemática de la aplicación del Derecho.

Durante mucho tiempo se identificó el contenido de la Ciencia Económica con el estudio de un mecanismo de interacción específico: el mercado. También la Ciencia Jurídica -por lo menos en la Europea continental- se ha limitado hasta fechas muy recientes al análisis de la aplicación del Derecho, es decir, al estudio del funcionamiento de un mecanismo específico: el ordenamiento jurídico vigente.

En fechas recientes este servilismo respecto de un mecanismo específico ha sido abandonado en favor de un planteamiento más general en el que el mercado no es más que uno de los múltiples mecanismos de articulación entre la racionalidad individual y la colectiva. En el campo del Derecho y en nuestro país, trabajos como los de Atienza y Calsamiglia ${ }^{8}$ apuntan ya a la necesidad de una consideración instrumental de la legislación como mecanismo para alcanzar ciertos objetivos colectivos. El planteamiento aquí propuesto pretende enfatizar que la discusión necesaria sobre las preferencias sociales, su origen, su posible dependencia de las preferencias subjetivas de los individuos, su dependencia de criterios morales o religiosos debe separarse, para los propósitos del análisis teórico, de una actividad que los juristas deben reivindicar que consiste en la articulación de mecanismos jurídicos coherentes que consigan los objetivos definidos por esas prioridades sociales. Se puede admitir que la «intención del legislador» u objetivos últimos del sistema normativo puedan tener orígenes externos al Derecho, sea un parlamento o unos principios éticos. Pero debe competer a los juristas decidir cómo legislar, es decir, cómo traducir estas intenciones en normas que induzcan sobre individuos libres comportamientos compatibles con las preferencias sociales. Y como ha sucedido en Economía, lo más probable es que no tarden en destilar de estos análisis resultados que confirmen en teoría la presencia permanente de teoremas de imposibilidad y paradojas que ponen en evidencia que la falacia de la composición es una constante en todas las ciencias sociales. Para legislar no basta con una mera declaración de intenciones. El diseño de un mecanismo jurídico es algo más complejo.

Un ejemplo más puede ayudar a aclarar conceptos. Consideremos una situación como la descrita en la figura: dos vehículos, A y B, se mueven en las direcciones indicadas. Si ambos siguen a la misma

${ }^{8}$ M. Atienza, Razón práctica y legislación, Actas del Congreso de la IVR, Gottingen 1991, y A. Calsamiglia, «¿Debe ser la moral el único criterio para legislar?, Doxa, vol. 13, 1993. 
velocidad se producirá una colisión. Cada uno de los conductores tiene dos acciones posibles: parar o avanzar. De la acción de uno solo de los agentes no podemos inferir las consecuencias: el resultado final es consecuencia de la interacción de las dos decisiones. Detenerse le supone a A una molestia equivalente a 2.000 pesetas en cualquier caso. Pero avanzar le supone una pérdida de 100.000 pesetas si B decide hacer lo mismo (hay colisión) y ninguna pérdida en caso de que $\mathrm{B}$ decida detenerse. La situación de $\mathrm{B}$ es parecida: le supone una molestia equivalente a 1.000 pesetas detenerse. Pero avanzar le supone una pérdida de 100.000 pesetas si A decide hacer lo mismo y ninguna pérdida en caso de que A decida detenerse. En la figura 3 representamos la situación de los dos vehículos, y un cuadro esquemático que recibe el nombre de matriz de pagos: cada casilla representa las implicaciones para cada uno de los jugadores de un par de estrategias. Al decidir su acción A escoge la fila relevante y B escoge la columna. Se llama equilibrio de Nash (o solución del juego) a todo par de estrategias en las que cada agente está escogiendo su mejor acción dada la acción del otro. Pues bien, resulta que en este juego hay dos situaciones de equilibrio que son que uno de los dos se detenga y que el otro avance. El hecho de que existan dos equilibrios implica la necesidad de coordinarse para saber quién pasa primero. $\mathrm{Y}$ las indecisiones se pagan con accidentes.

Una solución posible consiste en diseñar un mecanismo de mercado y subastar el derecho de paso. En este caso la puja entre los dos conductores subiría hasta 1.000 pesetas, punto a partir del cual el conductor B se retiraría. A tendría el derecho de paso (por menos cantidad de la que estaría dispuesto a pagar) y las 1.000 pesetas recaudadas podrían utilizarse para compensar a B por su espera. No sólo hemos coordinado las decisiones de los agentes, sino que incluso hemos puesto en marcha mecanismos redistributivos para compensar por las pérdidas a uno de los individuos.
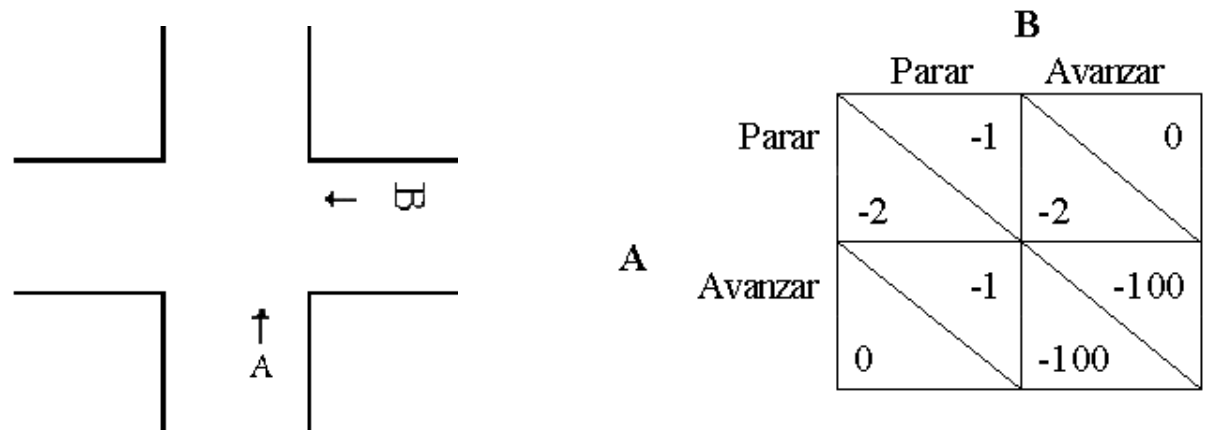

Figura 3 
Los costes de poner en práctica este mecanismo en cada cruce de carretera es tan inmenso que, en general, se ha recurrido a otros tipos de mecanismos de coordinación. La preferencia social en este caso es evidentemente evitar accidentes (en caso de que los conductores no se pongan de acuerdo y decidan avanzar simultáneamente) o paradas innecesarias (lo que puede ocurrir si suponiendo que el otro no va a detenerse ambos deciden parar). En efecto, ambas situaciones son ineficientes (en el sentido de Pareto, es decir, son dominadas por otras situaciones que son unánimemente preferidas).

Un mecanismo alternativo al mercado sería proponer una norma general y abstracta del tipo «tienen preferencia de paso los vehículos que vienen por la derecha». Supongamos que la sanción impuesta al que no cumpla la norma es de 10.000 pesetas. Ahora el conductor B tiene derecho de paso y A la obligación de detenerse. Pero siempre existe la posibilidad de que A decida no cumplir la ley. Una ley bien diseñada hará que esté en el interés de A cumplir la norma. Observemos, en primer lugar, que lo que en el esquema formal anterior llamamos la función $\varphi$ viene representado en nuestro ejemplo por la matriz de pagos que indica las consecuencias para cada agente de cada par de acciones. Tenemos también en este ejemplo unas preferencias sociales que indican que hay que evitar las casillas superior izquierda e inferior derecha -que son ineficientes- y escoger una de las dos restantes (que son óptimos de Pareto). El diseño de un sistema normativo es algo más complicado que la mera declaración de intenciones del tipo «se prohíbe tener accidentes». La norma establece una prioridad de paso arbitraria (de hecho totalmente insensible a consideraciones económicas que aconsejaban dar prioridad al conductor A que en algún sentido «tenía más prisa») y una sanción en el caso de infracción. En realidad lo que ocurre es que la norma modifica la matriz de pagos con la que se enfrentan los dos conductores, es decir, modifica la función $\varphi$ que asocia acciones y consecuencias. La nueva matriz de pagos será:

\section{B}

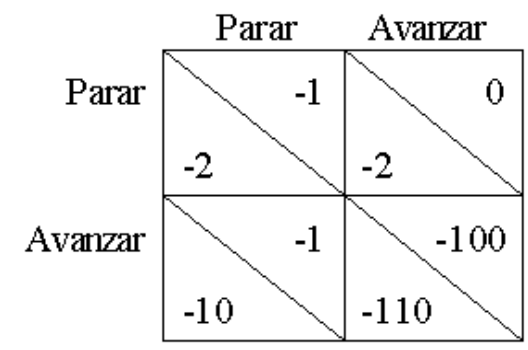

Figura 4 
Lo único que ha cambiado de un caso a otro es que si A decide hacer caso omiso de la norma deberá pagar una multa de 10.000 pesetas (por lo que en caso de que B también decida avanzar su pérdida será de 110.000 pesetas). ¿Cuál es la solución de este juego? El conductor B razona del modo siguiente: si A se detiene yo perderé 1.000 pesetas si también me detengo y no perderá nada si sigo avanzando. Por tanto, si A se detiene lo que me conviene es avanzar. En cambio, si A no se detiene perderé 1.000 pesetas si me detengo y perderé 100.000 pesetas si decido avanzar. Por tanto, lo que me conviene hacer si A no se detiene es parar. En otras palabras, lo que le conviene hacer es lo contrario de lo que haga A. Está en la misma situación de perplejidad que antes.

El razonamiento de A es distinto. Si B es prudente y decide parar aunque no se lo exija la ley -piensa- perderé 2.000 pesetas si paro o 10.000 pesetas si no paro (que es el importe de la multa). Entre perder 2 ó 10 prefiero perder 2: en caso de que B decida parar lo que me conviene es parar. Si B, haciendo uso de su prioridad de paso decide no detenerse entonces perderé 2.000 pesetas si me detengo y 110.000 pesetas si no me detengo. Resulta pues que para A detenerse es ahora una estrategia dominante: haga lo que haga $B$ lo que te interesa es detenerse. Por tanto A se detendrá y en este caso lo que le conviene a $\mathrm{B}$ es no parar. El juego tiene ahora una solución única y Pareto óptima.

Lo importante de este ejemplo es poner de manifiesto el modo indirecto en que la norma incide en el comportamiento de los agentes. Muchos aspectos adicionales deberán tenerse en cuenta a la hora del diseño legislativo entendido como la mera articulación entre los intereses privados y los colectivos. Toda norma se inserta en un sistema jurídico y no es obvio cómo diseñar los mecanismos respetando ciertas condiciones de consistencia, plenitud y economía.

La legalización de la droga constituye un buen ejemplo. Muchos reducen la discusión al terreno de la moral o de los derechos. Lo que hemos tratado de decir aquí es que incluso si se admite que el consumo de drogas es algo no deseable socialmente y que debe evitarse (es decir, si estamos de acuerdo en los objetivos sociales), muchos argumentan que la prohibición de la droga genera muchas más acciones delictivas y mucho más consumo de droga y que su legalización contribuiría a reducir su indeseable consumo: la prohibición genera un mercado de tráfico de drogas tan rentable que los efectos finales son peores. No sostengo que el argumento sea correcto o no, pero sí defiendo que un correcto diseño legislativo no puede dejar estas cuestiones sin responder. 


\section{Conclusiones}

La Economía y el Derecho son ciencias sociales que pueden tener en común mucho más de lo que tienen. En el presente trabajo me he esforzado en dar una visión algo distinta de la habitual de cómo estas disciplinas pueden influirse.

En primer lugar, la relación entre ambas disciplinas debe ir bastante más allá de la mera reducción de los fenómenos a dinero. Es cierto que buena parte de la Economía utiliza los precios y las correspondientes valoraciones monetarias como criterio último de valoración social. Algo tan cualitativo, diverso y difícilmente cuantificable como las preferencias individuales (que en principio pueden atender a consideraciones de tipo ético, religioso, altruista, además de las puramente económicas) se reducen a números fácilmente comparables. Que dichas comparaciones cuantitativas correspondan a algún criterio de deseabilidad social bien definido es precisamente lo que establece el teorema de la Mano Invisible. Pero, como hemos insistido, este teorema tiene un ámbito de aplicación limitado debido a los fallos del mercado, de un lado y al hecho de que la eficiencia paretiana es un criterio de deseabilidad social muy poco satisfactorio por ser totalmente insensible a cualquier definición aceptable de justicia o equidad, de otro. El tipo de interacción entre la Economía y el Derecho que postulamos tiene un carácter mucho más metodológico.

Como es típico en los modos de abstracción de los economistas he recurrido a hipótesis. En particular, he supuesto que los grandes objetivos sociales y su legitimación eran dados -dejando de lado toda la problemática de la Elección Social- y que el Derecho es algo dado y ya construido -dejando de lado también todas las aportaciones de autores como Dworkin y Calabresi-. Naturalmente, eso no son más que hipótesis de trabajo que me permiten diseccionar el objeto de estudio para analizarlo ${ }^{9}$. Esta reducción del universo de discurso me ha permitido plantear el Derecho como un mecanismo de coordinación, como un instrumento de articulación entre la racionalidad individual y la colectiva.

El paralelismo con el enfoque de la moderna Teoría Económica del Bienestar es evidente. En ambos casos se trata de un planteamiento fundamentalmente prescriptivo en el cual los mismos mecanismos de coordinación económica o jurídica se constituyen en objetos de elección. Esta elección se realiza en términos de la medida en

${ }^{9}$ Consciente, claro está, de que la disección -al separar brutalmente cosas que van indisolublemente unidas- acaba muchas veces con la vida del ser estudiado. Pero no por ello deja de ser un procedimiento imprescindible para el análisis. 
que mecanismos alternativos permiten alcanzar ciertos objetivos sociales. Aparecen entonces dos campos de análisis.

El primero se centra, como ha ocurrido en Economía, en el sentido que pueda tener la racionalidad colectiva y de qué consideraciones depende la formación de preferencias colectivas que son las que definen la intención del legislador. Estaríamos hablando de la racionalidad ética del Derecho.

El segundo, al que hemos dedicado aquí nuestra atención de modo preferente en este trabajo, tiene que ver con lo que Bobbio ${ }^{10}$ llama racionalidad fuerte o adecuación de medios a fines en la creación de legislación, por contraposición a una razón débil que se limita a aplicar a casos concretos las reglas establecidas. Tomando las preferencias sociales como un dato, se trata de diseñar los mecanismos jurídicos que induzcan a los individuos a comportarse de forma que se alcancen los fines especificados en las preferencias sociales.

$\mathrm{Ni}$ en Economía ni en Derecho nos limitamos ya a contemplar el mundo como la plasmación de unos valores y de una lógica que se revelan y se imponen a la mente humana de la misma manera que se creía que lo hacían las leyes de la naturaleza. Por el contrario, nuestras sociedades son el resultado de decisiones acerca de los mecanismos de interacción, de las instituciones y de los sistemas jurídicos, cuyo diseño y mejora permanente debe ser siempre el objetivo último de economistas y juristas.

El éxito de la gran labor de ingeniería social que nos toca a los economistas y a los juristas de hoy para diseñar nuestra sociedad no depende sólo del ardor con el que defendamos los grandes ideales sociales, sino también del empeño y el rigor con el que nos apliquemos a identificar y sortear todas las dificultades de tipo informacional y motivacional que se interponen en el funcionamiento de nuestras instituciones.

${ }^{10}$ N. Bobbio, «La razón en el Derecho», Doxa, vol. 2, 1985. 